\title{
TOTAL GRAPHS AND TRAVERSABILITY
}

\section{by MEHDI BEHZAD AND GARY CHARTRAND $\dagger$}

(Received 22nd March 1966)

With every graph $G$ (finite and undirected with no loops or multiple lines) there is associated a graph $L(G)$, called the line-graph of $G$, whose points correspond in a one-to-one manner with the lines of $G$ in such a way that two points of $L(G)$ are adjacent if and only if the corresponding lines of $G$ are adjacent. This concept was originated by Whitney (3). In a similar way one can associate with $G$ another graph which we call its total graph and denote by $T(G)$. This new graph has the property that a one-to-one correspondence can be established between its points and the elements (the set of points and lines) of $G$ such that two points of $T(G)$ are adjacent if and only if the corresponding elements of $G$ are adjacent (if both elements are points or both are lines) or they are incident
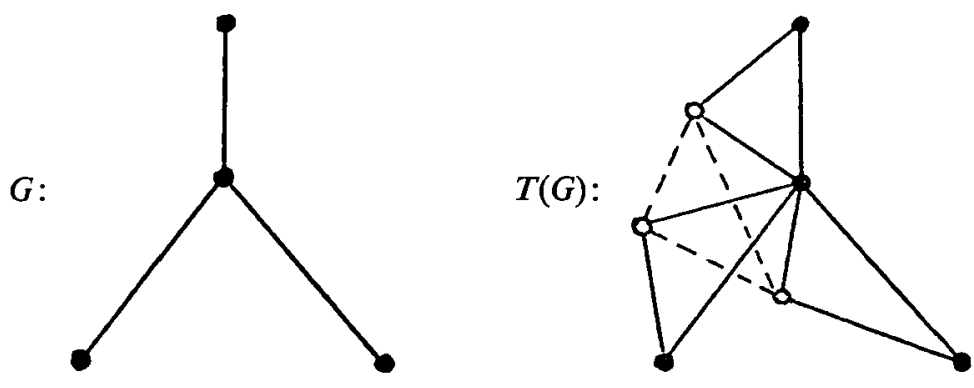

FIGURE 1

(if one element is a point and the other a line). In Fig. 1, a graph $G$ and its total graph $T(G)$ are shown. In $T(G)$ the " dark" points correspond to the points of $G$ while the "light" points correspond to the lines of $G$. The linegraph $L(G)$ is a subgraph of $T(G)$ and consists of the "light" points and the lines of $T(G)$ joining two such points. These lines are drawn in Fig. 1 with dashed lines.

In this note we present characterisations of graphs having Hamiltonian total graphs and of graphs whose total graphs are Eulerian. In addition, we prove that for every non-trivial connected graph $G, T(T(G))$ is Hamiltonian.

A graph $G$ is Hamiltonian if it has a Hamiltonian cycle, i.e. a cycle containing all points of $G$. (For a discussion of these graphs, the reader is directed to

$\dagger$ Work supported by the U.S. Air Force Office of Scientific Research under grant AF-AFOSR-754-65. 
Ore (2).) A necessary and sufficient condition for a graph whose total graph is. Hamiltonian is presented in our first theorem.

Two elements $a$ and $b$ of $G$ are said to be neighbours if one of the following holds: (i) $a$ and $b$ are adjacent points, (ii) $a$ and $b$ are adjacent lines, (iii) one of $a$ and $b$ is a point and the other an incident line. A graph is trivial if it consists of a single point.

Theorem 1. The total graph $T(G)$ of a non-trivial graph $G$ is Hamiltonian if and only if the set of all elements of $G$ can be ordered in such a way that consecutive elements are neighbours as are the first and last elements.

Proof. If the elements of $G$ can be so appropriately ordered, then by taking the corresponding points of $T(G)$ in this same order, a Hamiltonian cycle is produced. Conversely, if $T(G)$ contains a Hamiltonian cycle

$$
\left(V_{0}, V_{1}, \ldots, V_{n-1}, V_{n}=V_{0}\right) \text {, }
$$

then let $a_{i}$ be that element of $G$ associated with the point $V_{i}$. The ordering $a_{0}, a_{1}, \ldots, a_{n-1}, a_{n}=a_{0}$ has the desired properties.

Theorem 2. A sufficient condition for $T(G)$ to be Hamiltonian is that $G$ be Hamiltonian.

Proof. Assume $G$ has $p$ points and $q$ lines, and let $C=\left(V_{0}, V_{1}, \ldots, V_{p}=V_{0}\right)$ be a Hamiltonian cycle of $G$. We construct an ordered sequence of the $p+q$ elements of $G$ such that consecutive elements are neighbours and the last element is a neighbour of the first, as follows: Let $V_{0}$ be the first element of the sequence, and then select in any order all lines of $G$ incident to $V_{0}$ which are not in $C$ (if any), followed by the line $V_{0} V_{1}$ of $C$ and the point $V_{1}$. Next in the sequence, choose those lines (if any) which are incident to $V_{1}$ and not in $C$, followed by the line $V_{1} V_{2}$ of $C$ and the point $V_{2}$. This process is repeated, except that lines not in $C$ are omitted in the ordering if they have been previously included in the sequence. By this procedure, the line $V_{p-1} V_{p}$ of $C$ is obtained as the final element of this sequence, and this line is a neighbour of $V_{0}$. Thus by Theorem 1 , $T(G)$ is Hamiltonian.

By way of notation, we let $T^{2}(G)$ denote $T(T(G))$ and, in general,

$$
T^{n}(G)=T\left(T^{n-1}(G)\right)
$$

for $n \geqq 2$, where $T^{1}(G)=T(G)$. Using induction and the result of Theorem 2 , we obtain an immediate corollary.

Corollary 2a. If $G$ is Hamiltonian, then $T^{n}(G)$ is Hamiltonian for all $n \geqq 1$.

In a Hamiltonian graph all its points can be traversed with a single cycle. If a connected graph has the property that each of its lines can be traversed exactly once with a single closed path in which points may be encountered more than once, then the graph is called Eulerian. It is well known that a graph is Eulerian if and only if it is connected and every point has even degree, i.e. the number of incident lines with each point is even. 
The following observation will prove useful in our next theorem.

Remark. If $V$ is a point of $G$ and $V^{1}$ is the corresponding point of $T(G)$, then the degree of $V^{1}$, denoted by $\operatorname{deg} V^{1}$, equals $2 \operatorname{deg} V$, while if $X=V_{1} V_{2}$ is a line of $G$ and $X^{1}$ is the corresponding point of $T(G)$, then

$$
\operatorname{deg} X^{1}=\operatorname{deg} V_{1}+\operatorname{deg} V_{2} .
$$

Theorem 3. Let $G$ be a connected graph. Then $T(G)$ is Eulerian if and only if all points of $G$ are of the same parity.

Proof. By the parity of point we mean the parity of its degree. Let $T(G)$ be an Eulerian graph, and assume, to the contrary, that $G$ has a point $V_{1}$ with odd degree and a point $V_{2}$ with even degree. Since $G$ is connected, $V_{1}$ and $V_{2}$ are joined by a path on which exist two adjacent points $V_{3}$ and $V_{4}$ of opposite parity. Thus, by the preceding remark, the point in $T(G)$ which corresponds to the line $V_{3} V_{4}$ has odd degree, which contradicts the fact that $T(G)$ is Eulerian.

The converse is a direct consequence of the remark.

Corollary 3a. If $G$ is Eulerian, then $T(G)$ is Eulerian.

Even if all points of a connected graph are not of the same parity, then $T(G)$ contains an Eulerian subgraph of a special type. A subgraph $H$ of a graph $G$ is a spanning subgraph if it contains all the points of $G$.

Theorem 4. The total graph $T(G)$ of every connected graph $G$ contains $a$ spanning Eulerian subgraph.

Proof. The result is obvious if $G$ consists of a single point. Otherwise, the connected spanning subgraph $H$ of $T(G)$ obtained by deleting all lines of the line-graph $L(G)$ is Eulerian since all its points have even degree.

Theorem 5. If a non-trivial graph $G$ contains a spanning Eulerian subgraph, then $T(G)$ is Hamiltonian.

Proof. Let $S$ be a spanning Eulerian subgraph of $G$. The lines of $S$ can be arranged in a cyclic fashion $C=\left(X_{0}, X_{1}, \ldots, X_{r-1}, X_{r}=X_{0}\right)$; we let $V_{i}$ be that point incident with the lines $X_{i}$ and $X_{i+1}, i=0,1, \ldots, r-1$. Of course, some of the points $V_{i}$ may be identical. We construct an ordered sequence of elements of $G$ such that consecutive elements are neighbours and the last element is a neighbour of the first as follows: Let $X_{0}$ be the first term of the sequence, $V_{0}$ the second, and then follow these by all lines of $G$ incident to $V_{0}$. which are not in $C$ (if any). These elements are followed by $X_{1}, V_{1}$, and all those lines of $G$ which are incident to $V_{1}$ and not in $C$ (if any). This procedure is continued except that lines and points are omitted from the sequence if they have been previously included in the sequence. Finally, the line $X_{r-1}$ of $C$ will be in the sequence followed by the point $V_{r-1}$ if it has not been previously taken. But in any case $X_{0}$ is a neighbour of both $V_{r-1}$ and $X_{r-1}$. By Theorem 1, $T(G)$ is Hamiltonian.

Corollary 5a. If $G$ is Eulerian, then $T(G)$ is Eulerian and Hamiltonian. 
It follows by Theorem 4 that if $G$ is a non-trivial connected graph, then $T(G)$ has a spanning Eulerian subgraph, which, according to Theorem 5, implies that $T(T(G)$ ) is Hamiltonian. This result is stated below.

Theorem 6. If $G$ is a non-trivial connected graph, then $T^{2}(G)$ is Hamiltonian.

Corollary 6a. If $G$ is a non-trivial connected graph, then $T^{n}(G)$ is Hamiltonian for all $n \geqq 2$.

This corollary serves as an analogue to the theorem in (1) which states that if $G$ is a connected non-trivial graph with $p$ points which is not a simple path, then the iterated line-graph $L^{n}(G)$ is Hamiltonian for all $n \geqq p-3$. The conclusion of Corollary 6a cannot, in general, be improved since, for example, the graph $G$ of Fig. 1 is non-trivial and connected but $T(G)$ is not Hamiltonian.

\section{REFERENCES}

(1) G. Chartrand, The existence of complete cycles in repeated linegraphs, Bull. Amer. Math. Soc. 71 (1965), 668-670.

(2) O. ORE, Theory of Graphs (Providence, 1962).

(3) H. WhitNey, Congruent graphs and the connectivity of graphs, Amer. J. Math. 54 (1932), 150-168.

Wayne State University AND

PAHLAVI UNIVERSITY, IRAN

THE UNIVERSITY OF MiChIGAN AND

WESTERN MICHIGAN UNIVERSITY 